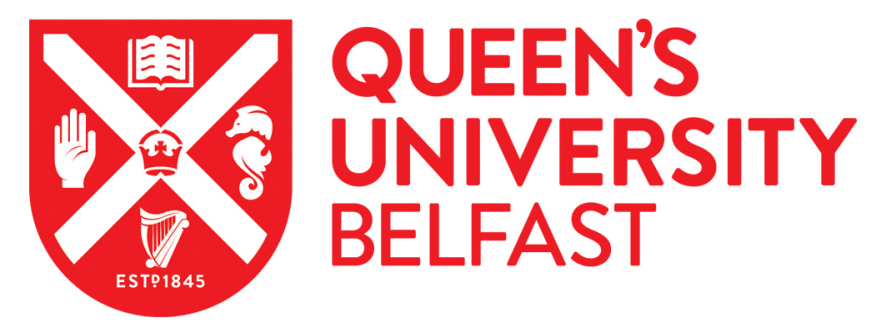

\title{
Application of a mechanobiological simulation technique to stents used clinically
}

Boyle, C. J., Lennon, A. B., \& Prendergast, P. J. (2013). Application of a mechanobiological simulation technique to stents used clinically. Journal of Biomechanics, 46(5), 918-924.

https://doi.org/10.1016/j.jbiomech.2012.12.014

\section{Published in:}

Journal of Biomechanics

\section{Document Version:}

Peer reviewed version

Queen's University Belfast - Research Portal:

Link to publication record in Queen's University Belfast Research Portal

\section{Publisher rights}

(C) 2013 Elsevier. Licensed under the Creative Commons Attribution-NonCommercial-NoDerivatives 4.0 International

$\mathrm{http}: / /$ creativecommons.org/licenses/by-nc-nd/4.0/ which permits distribution and reproduction for non-commercial purposes, provided the author and source are cited.

\section{General rights}

Copyright for the publications made accessible via the Queen's University Belfast Research Portal is retained by the author(s) and / or other copyright owners and it is a condition of accessing these publications that users recognise and abide by the legal requirements associated with these rights.

\section{Take down policy}

The Research Portal is Queen's institutional repository that provides access to Queen's research output. Every effort has been made to ensure that content in the Research Portal does not infringe any person's rights, or applicable UK laws. If you discover content in the Research Portal that you believe breaches copyright or violates any law, please contact openaccess@qub.ac.uk. 


\section{Application of a Mechanobiological Simulation Technique to Stents used Clinically}

Colin J. Boyle1, Alex B. Lennon ${ }^{1,2}$, Patrick J. Prendergast1,*

${ }^{1}$ Trinity Centre for Bioengineering, School of Engineering, Trinity College Dublin, Ireland

${ }^{2}$ School of Mechanical and Aerospace Engineering, Queen's University Belfast, Northern Ireland

*email: pprender@tcd.ie

Submitted to the Journal of Biomechanics. 


\section{Abstract}

Many cardiovascular diseases are characterised by the restriction of blood flow through arteries. Stent implantation is increasingly used to treat blockages; however, tissue in-growth into the stent can lead to failure of this treatment (restenosis). In order to predict the long-term efficacy of this treatment, a mechanobiological model of the arterial tissue reaction to stress may be required. In this study, a computational model of arterial tissue response to stenting is applied to three clinicallyrelevant stent designs. We ask the question whether such a mechanobiological model can differentiate between stents as found clinically, and we compare these predictions to a purely mechanical analysis. In doing so, we are testing the hypothesis that mechanically-induced injury to arterial tissue stimulates restenosis.

Finite element analysis of the expansion of three different stent types was performed in an idealised, 3D artery. Injury was calculated in the arterial tissue using a remaining-life damage mechanics approach. The inflammatory response to this initial injury was modelled using ordinary differential equations governing variables which represented tissue-degrading species and growth factors. Three levels of inflammation response were modelled to account for inter-patient variability. A latticebased model of Smooth Muscle Cell behaviour was implemented, treating cells as discrete agents governed by local rules.

The study found that the model predicted differences between stent designs similar to those found in vivo. It showed that the volume of neointima produced could be quantified, providing a quantitative comparison of stents. In contrast, the differences between stents based on stress alone were highly dependent on the choice of comparison variables. Higher prescribed inflammation response was found to reduce the differences between stents.

This study shows that mechanobiological modelling may provide a valuable tool in stent design, allowing predictions of their long-term efficacy. The level of inflammation was shown to affect the sensitivity of the model to stent design. If this finding were verified in patients, this could suggest that high-inflammation patients may require alternative treatments to minimise restenosis. 


\section{Introduction}

Many cardiovascular diseases are due to the blockage of arteries due to plaque build-up. Removing blockages to restore blood flow by implanting a cardiovascular stent is quite successful, except that a long-term adaptive (and/or healing) response of the arterial tissue can lead to restenosis, which is a re-blockage of the artery due to tissue ingrowth through the stent into the lumen of the vessel. In order to design stents to optimise long-term lumen gain, a mechanobiological model of arterial tissue adaptation is necessary.

Restenosis has been correlated to injury and inflammation (Gunn et al., 2002; Kornowski et al., 1998), and a paradigm of wound-healing has been adopted to explain restenosis progression (Farb et al., 2007; Geary et al., 1998; Welt \& Rogers, 2002). The restenotic response begins immediately upon stent expansion, when damage is imparted to the arterial tissue. The tissue is stretched circumferentially, and compressed radially beneath the stent struts (Gunn et al., 2002). Cell death and tissue tearing may occur, and the endothelium is denuded through the shearing force of the stent and expanding balloon. These injuries induce a healing response from the arterial tissue (Boyle et al. 2010). Inflammatory cells, such as leukocytes and monocytes, attach to the injured regions from the blood, and infiltrate the damaged tissue. Matrix degrading proteins (such as matrix metalloproteintases) are expressed, which enzymatically degrade the collagen and other tissue constituents in order to remove damaged tissue. Growth stimuli are released from the degrading tissue, from the inflammatory cells, from the blood, and from apoptotic/necrotic smooth muscle cells (SMCs). The degradation of extracellular matrix has the effect of stimulating a phenotype modulation in the SMCs; this means that the SMCs alter their phenotype, losing their ability to contract, becoming synthetic, proliferative and matrix synthesising. These synthetic SMCs are the cells which produce the neointimal tissue which leads to restenosis. The SMCs can return to a 
contractile phenotype once the extracellular environment is restored to the physiological state (Thyberg, Hedin, Sjolund, Palmberg, \& Bottger, 1990).

Several studies have attempted to optimise or evaluate stent design in relation to arterial wall mechanics. Bedoya et al. (2006) and Timmins et al. (2007) optimised stent designs by minimising the volume of tissue stressed above a critical threshold. This approach attempts to capture restenosis by predicting the regions where adverse biological reactions are likely to occur. However, the link between stress and tissue growth is not so simple and a mechanobiological model is needed to predict the outcome of these adverse reactions. Lally \& Prendergast (2006) were the first to attempt a predictive model of restenosis based on the tissue injury induced by stent expansion. In that study, a finite element model was implemented in which the accumulation of damage was assumed to induce tissue in-growth into the arterial lumen; however, the model could not predict stabilization of the lesion because of the rudimentary modelling of cell activity. The technique was also deterministic, and could not account for the intrinsic stochastic nature of biological processes, or the variation in patient responses to injury. Stabilization of a simulated restenosis was recently achieved using a stochastic cell-centered mechanobiological model driven by mechanically induced injury (Boyle et al. 2010),

In a previous study (Boyle et al, 2011), we developed, calibrated, and tested a cell-centred, lattice-based model, which modelled the injury, inflammation and biological response of arterial tissue to stent and angioplasty induced injury. This technique models SMC behaviour by explicitly modelling individual cells, which interact with their local environment and neighbours within a regular orthogonal lattice (Pérez \& Prendergast, 2007). Cells migrate, proliferate, differentiate and produce extracellular matrix according to rules, which allows the implementation of known cell behaviour (for a review of SMC behaviour, see Thyberg et al. (1990)). In order to test the suitability of using such a mechanobiological model as an engineering design tool, the predictions of the model must be compared to in vivo, clinical data. Hoffmann et al. (2001) performed a detailed study of the 
clinical performance of three different stent designs, which included information on the stent types, and detailed data on the outcomes of these stents in terms of the neointimal areas produced. Kastrati et al. (2001) also analysed clinical data for the same stent types, and ranked their performance based on a multivariate analysis of restenosis predictors. Both of these studies showed the MULTILINK stent (a flexible, open-cell design) to perform better than both the PALMAZ stent (a stiffer, slotted tube design) and the NIR (a closed-cell design).

In this study, we set out to test the hypothesis that injury mechanobiological model of arterial tissue response to injury, as developed and calibrated previously, could predict the longterm outcomes of stent design. We applied the simulation technique in 3D to three stents similar in design to the ones analysed clinically by Hoffmann et al. (2001) and Kastrati et al. (2001). If the hypothesis is corroborated, then this methodology could be applied to future device design and treatment options. It may also prove useful in identifying patients who may be susceptible to restenosis with a given device design, allowing the patient-specific and lesion specific matching of stents.

\section{Methods}

The simulation technique used in this study has been described in detail in a previous paper (Boyle et al. 2011). The technique uses three interconnected models: 1 . An injury model, where a finite element model of stent expansion, coupled with damage accumulation, allows the calculation of injury in the artery. 2. An inflammation model, in which tissue degradation and growth factor production in response to injury, and the removal of that injury, is modelled using ODEs. 3. A latticebased model of SMC activity, which captures the phenotype modulation, proliferation, migration and tissue production of SMCs. The technique was adapted to 3D, and applied to three different stent designs. 


\section{Injury model}

The artery was modelled as a cylindrical vessel, with isotropic, hyperelastic material properties and inner and outer radii of $1 \mathrm{~mm}$ and $2 \mathrm{~mm}$ respectively. A Mooney-Rivlin formulation was used and calibrated to the data of Lally et al. (2005), who measured the stretch response of porcine coronary artery to uniaxial tensile stress. The injury in the arterial tissue was calculated using the von Mises stress criterion and a remaining life approach, in which the damage is related to the number of cycles undertaken during a loading period versus the number of cycles to failure from fatigue test. A log-linear relationship was calibrated to fatigue data for porcine coronary arterial tissue (from McLoughlin 2008):

$\sigma=\alpha \log N_{f}+\sigma_{f}$

Where $\alpha$ is a constant, $\sigma_{\mathrm{f}}$ is the failure strength of the material based on a uniaxial test, and $N_{f}$ is the number of cycles to failure. Damage was assumed to accumulate according to Miner's rule, with a rate of

$\dot{D}=\frac{1}{N_{f}}$

Assuming the material has a fatigue limit $\left(\sigma_{0}\right)$, an equation for damage accumulation as a function of stress can be determined:

$$
\begin{aligned}
& D=10 \frac{\sigma_{f}-\sigma}{\alpha} \quad \sigma_{0}<\sigma_{\mathrm{I}} \leq \sigma_{f} \\
=0 & \sigma \leqq \sigma_{0} \\
=1 & \sigma>\sigma_{f}
\end{aligned}
$$

\section{Inflammation model}

The inflammatory response was modelled as two variables: a matrix degrading factor (MDF), and a growth factor (G). Extracellular matrix (ECM) was explicitly modelled as a variable with a value from zero (no ECM present) to one (fully dense ECM). The presence of MDF stimulates the removal of 
ECM within the injured region, and G controls the proliferation of SMCs. The production of MDF is a function of the amount of injury present (assumed to be linear in this implementation), and the MDF reacts with the ECM by reducing both components at a rate proportional to the MDF present (i.e. a first order reaction).

These variables are modelled on a regular orthogonal lattice, which also holds the SMCs. The parameters for the differential equations governing these species were calibrated to the data of Cipollone et al. (2001). In that study, the levels of a marker for inflammation (monocyte chemoattractant protein-1) were monitored in the blood of patients after coronary angioplasty. It was found that those patients who went on to develop restenosis had higher MCP-1 levels over a longer period than those that did not. We established three responses based on that data to capture the variability inherent in a population: low inflammation corresponding to the group which expressed lower MCP-1, high inflammation corresponding to the group which expressed high MCP1, and an intermediate response, which lay half-way between the high and low response curves. Further details can be found in Boyle et al. (2011).

\section{Smooth muscle cell model}

The phenotype of SMCs is a function of the amount of ECM present in its local region, with high ECM inducing a contractile phenotype (the relationship is assumed to be linear in this implementation). This phenotype governs the maximum proliferation and migration rates within the tissue. The actual probability of proliferation of a cell is dependent on the availability of suitable neighbour sites, and the amount of growth stimulus in the region. The growth stimulus decays exponentially over time.

Due to the size of the artery and the average size of SMCs an impractically large number of lattice points would have been required to run a full-scale simulation. Instead, a coarse-graining approach was applied, in order to achieve a practical solution time. The lattice used had a characteristic length (point spacing) of $0.05 \mathrm{~mm}$, compared to $0.01825 \mathrm{~mm}$ in the previous model. In this way, a scalingup of the lattice was performed, whereby each lattice cell represented several biological cells, in a 
mesoscopic representation of the cells. The lattice cells remain independent random walking cells, and all parameters are conserved from the 2D model, but are scaled accordingly. In particular, the migration rate of the cells is related to lattice spacing, as is the initial amount of lattice cells present ${ }^{1}$.

\section{Stent Geometries}

Three stent types were modelled: a design similar to the commercially available MULTILINK stent, a design similar to the NIR stent, and a design similar to the PALMAZ stent (Figure 1). The material model used for the stent was an elasto-plastic model. Each stent was expanded to a balloon:artery ratio of 1.2. Finite element simulations were solved with ABAQUS/Explicit, using a quasi-static analysis.

The simulations were performed on each of the three stents using parameters calibrated to high, low and intermediate inflammatory responses to injury, as outlined above. Each stent/inflammation combination was simulated with three replicates, producing 27 simulation runs overall.

To generate results, the cross-sectional areas of lumen and neointima were measured at several axial sections along the axis of the artery. The total number of cells and total volume of tissue was recorded during the simulation.

The simulations were implemented using the MechanoBiology ToolKit (MBTK), which is a library built upon VTK (the Visualization ToolKit, Kitware Inc., USA) to produce mechanobiological models using lattice-based methods (Lennon et al. 2011). Finite Element Analysis was performed using Abaqus v6.8 (Simulia).

\footnotetext{
${ }^{1}$ For example, $v_{\text {SMC,lattice }}=v_{\text {SMC }} \frac{\boldsymbol{\Delta} t}{\boldsymbol{\Delta} x}$ is the scaled cell migration speed in lattice points per increment, as a function of the time and space increments and the real migration speed. For cell numbers, the fraction of occupied lattice points is kept constant.
} 


\section{Results}

The finite element analysis predicted similar peak von Mises stresses for each stent - within a range of $0.7 \mathrm{MPa}$. The NIR induced the highest peak von Mises stresses at $4.0 \mathrm{MPa}$, followed by the PALMAZ at 3.9 MPa and the MULTILINK at 3.3 MPa;. Stresses were highest around the stent struts, and the highest of these were at stent crowns. With the MULTILINK, the highest stresses were induced at the stent ends.

The injury induced at $80 \%$ expansion shows a greater amount of injury present in the PALMAZ and NIR stents compared to the MULTILINK (indicated by the grey regions in Figure 2), and this persists at full expansion. Peak stresses upon removal of the balloon and stent recoil were $1.30 \mathrm{MPa}$ for the NIR stent, 0.33 MPa for the PALMAZ stent, and 0.3 MPa for the MULTILINK stent.

The simulation produced a large amount of restenosis in the three stents (Figure 3). A gradual production of restenosis is predicted within the stent area, eventually covering the stent, and reducing lumen area (Figure 4). The simulation predicts a gradual reduction in the amount of active cells in the neointimal area, and predicts a relatively acellular final neointimal area, whereas in the media and deep neointima, cells accumulated. Cell numbers in the model at the end-point of the simulations were higher for the NIR than the PALMAZ, and both were higher than the MULTILINK stent (Figure 5).

The neointimal areas in the MULTILINK stent produced lower restenosis rates when simulation runs were analysed (Figure 6); there was little difference found along the length of the stent between the PALMAZ and the NIR. The extent of the differences between stents was dependant on the inflammation present with differences more pronounced in the low inflammation case.

The volume of tissue above several Von Mises stress thresholds was calculated for each stent. The differences between stents was then evaluated as the ratio of tissue above the threshold for each stent. For each stress criterion used, the PALMAZ and NIR stents achieved similar results (with the 
greatest difference occurring when the damage limit (1.417 $\mathrm{MPa}$ ) was used). The difference between these two stents and the MULTILINK stent depended on the level of the stress criterion used, with the differences greater for higher stress thresholds (Error! Reference source not found.).

The neointimal volume within the stents depended on the level of inflammation, with low and intermediate responses producing a higher growth rate at early time points than the high response (shown for MULTILINK stent in Figure 7). The amount of restenosis at 240 days was inversely related to the initial speed of lesion growth, and directly related to inflammation level. This data also shows that the rate at which quiescence (a low growth rate) was achieved was inflammation dependent; the MULTILINK, for example, becomes quiescent for low inflammation at approximately 150 days (Figure 7). The predictions of the relative efficacies, measured as the ratio of the numbers of cells produced between stents, were different depending on the amount of inflammation (Figure 8). Furthermore, relative efficacy of the PALMAZ and NIR stents also altered over time, and was related to the inflammation level (Figure 8). In contrast, the PS and NIR responses were almost identical to each other so that their relative efficacy converged to the same value (Figure 8).

\section{Discussion}

In this paper, we tested the hypothesis that a mechanobiological model of arterial tissue response to injury could predict the long-term restenosis outcomes of clinical stent designs. The simulation technique predicted the same ranking of stents based on average neointimal area as two clinical studies (Hoffmann et al., 2001; Kastrati et al., 2001) with the open-cell-type stent outperforming more rigid stents. Simulations predicted less of a difference between the PALMAZ and NIR stent than between the MULTILINK and PALMAZ stent, in terms of both tissue volume and cell numbers. The magnitude of the differences between the stent designs was greater when considering cell numbers; these results closely matched not only the ranking but the quantitative difference and variability observed in the clinical trial (Error! Reference source not found.), and for this reason we believe the hypothesis posed has been corroborated. 
The clinical study of (Hoffmann et al., 2001) showed large variations in the neointima formed in the stents, so much so that a statistically significant difference in neointimal area along the axis of the stent was not found between the stents. Variability in our study was due to the stochastic nature of cell activities. The simulations described here showed a similar trend to the clinical studies when the stents are compared using cell numbers produced. However, the differences between the NIR and PALMAZ in the model were not as pronounced as between the corresponding designs in the Hoffmann study (the geometry of the stent used in this study was much simpler than the INFLOW design used in the clinical study). A limitation of this study is that the model described here may not account for some of the differences between stent designs. In particular, many corrugated ring stents can have a more rounded cross-section strut, while the PALMAZ stent is rectangular. This may have an important part to play in cell migration around the stent. The number of crowns in the stents was constant for all three stents modelled here, but this may not be the case for stents used clincially.

The variability observed in clinical studies may come from the inherent variability between patients in terms of lesion size, biological response, procedural variability, etc., which were not included in the computational model developed in this study. The differences between the stents may be more pronounced when considering patient-specific, complex lesion geometries and material properties, differences which may not show up in the straight, idealised artery model used here. The relatively small differences in neointima predicted between stents may, however, be close to the clinical reality, as several papers on the relative performance have pointed out that significant differences between current stent designs have been difficult to identify (Edelman \& Rogers, 1999; Windecker \& Meier, 2001).

The study described here did not include some important characteristics of stent implantations in clinical practice. There was no variability in the geometry or material properties of the host vessel, which was idealised, isotropic and homogeneous. This is important for gaining an 
accurate prediction of injury, but also to be able to predict the initial biological configuration of the artery. For example, the plaques of most arteries can have a-cellular regions, or regions with foam cells, and not SMCs. The distribution of SMCs was assumed to be constant in the arteries. Furthermore, the stent geometries and expansion methods could be adapted to be more realistic.

This study builds upon previous attempts to simulate restenosis. Lally et al. (2006) modelled tissue growth using diffusion equations in response to mechanical damage. The use of continuum equations to govern cell behaviour proved limiting in simulating lesion progression and quiescence. In contrast, a more mechanistic approach to cell behaviour, such as the lattice modelling approach taken in this study, allows for greater experimental validation and predictive power, as isolated components of the model can be tested separately. In comparison to our previous study (Boyle et al. 2011), this study was applied to 3D arteries, and clinically relevant stent designs. This represents a first step in the direction of applying such models to medical devices as a pre-clinical design tool. In contrast to purely stress-based optimisation, this method can provide a quantitative comparison between stents. Stress-only comparisons can only hope to rank stents, based on biophysical stimuli as a surrogate for future tissue growth. In order to quantify that response, a mechanobiological link such as is proposed in this study is needed.

This study demonstrates the use of computational simulation techniques to predict the mechanobiological reaction of arteries to stent implantation. It offers a pre-clinical technique capable of including the patient's restenotic reaction to stenting, and offers scope for including interpatient variability in the reaction to injury. In particular, the results of this study suggest that the sensitivity of a patient to stent design may depend on their inflammatory phenotype. The technique can be adapted, with more complex mechanical models, to patient-specific lesions. 


\section{Acknowledgements}

This work is funded by a Science Foundation Ireland (SFI) Principal Investigator grant to Prof. Patrick Prendergast and by Medtronic, Galway, Ireland, and by an Enterprise Ireland grant to Dr. Caitrona Lally. 


\section{References}

Bedoya, J., Meyer, C. a, Timmins, L. H., Moreno, M. R., \& Moore, J. E. (2006). Effects of stent design parameters on normal artery wall mechanics. Journal of Biomechanical Engineering, 128(5), 757-65. doi:10.1115/1.2246236

Boyle, C. J., Lennon, A. B., Early, M., Kelly, D. J., Lally, C., \& Prendergast, P. J. (2010). Computational simulation methodologies for mechanobiological modelling: a cell-centred approach to neointima development in stents. Philosophical transactions. Series A, Mathematical, physical, and engineering sciences, 368(1921), 2919-35. doi:10.1098/rsta.2010.0071

Boyle, C. J., Lennon, A. B., \& Prendergast, P. J. (2011). In Silico Prediction of the Mechanobiological Response of Arterial Tissue: Application to Angioplasty and Stenting. Journal of Biomechanical Engineering, 133(8), 081001. doi:10.1115/1.4004492

Cipollone, F., Marini, M., Fazia, M., Pini, B., lezzi, A., Reale, M., Paloscia, L., et al. (2001). Elevated circulating levels of monocyte chemoattractant protein-1 in patients with restenosis after coronary angioplasty. Arteriosclerosis, thrombosis, and vascular biology, 21(3), 327. Am Heart Assoc.

Edelman, E. R., \& Rogers, C. (1999). Stent-versus-stent equivalency trials: Are some stents more equal than others? Circulation, 100(9), 896-898. Am Heart Assoc.

Farb, A., Sangiorgi, G., Carter, a J., Walley, V. M., Edwards, W. D., Schwartz, R. S., \& Virmani, R. (2007). Pathology of acute and chronic coronary stenting in humans. Circulation, 99(1), 44-52.

Geary, R. L., Nikkari, S. T., Wagner, W. D., Williams, J. K., Adams, M. R., \& Dean, R. H. (1998). Wound healing: a paradigm for lumen narrowing after arterial reconstruction. Journal of Vascular Surgery, 27(1), 96-108. Elsevier.

Gunn, J., Arnold, N., Chan, K. H., Shepherd, L., Cumberland, D. C., \& Crossman, D. C. (2002). Coronary artery stretch versus deep injury in the development of in-stent neointima. Heart, 88, 401-405.

Hoffmann, R., Jansen, C., König, a, Haager, P. K., Kerckhoff, G., vom Dahl, J., Klauss, V., et al. (2001). Stent design related neointimal tissue proliferation in human coronary arteries; an intravascular ultrasound study. European heart journal, 22(21), 2007-14. doi:10.1053/euhj.2001.2606

Kastrati, a, Mehilli, J., Dirschinger, J., Pache, J., Ulm, K., Schühlen, H., Seyfarth, M., et al. (2001). Restenosis after coronary placement of various stent types. The American journal of cardiology, 87(1), 34-9.

Kornowski, R., Hong, M. K., Tio, F. O., Bramwell, O., Wu, H., \& Leon, M. B. (1998). In-stent restenosis: contributions of inflammatory responses and arterial injury to neointimal hyperplasia. Journal of the American College of Cardiology, 31, 224-230. 
Lally, C., \& Prendergast, P. J. (2006). Simulation of In-stent Restenosis for the Design of Cardiovascular Stents. Mechanics of Biological Tissue (pp. 255-267). Springer-Verlag.

Lally, C., Dolan, F., \& Prendergast, P. J. (2005). Cardiovascular stent design and vessel stresses: a finite element analysis. Journal of Biomechanics, 38(8), 1574-81. doi:10.1016/j.jbiomech.2004.07.022

Lennon, A.B., Khayyeri, H., Xue, F., \& Prendergast, P. J. (2011). Biomechanical Modelling of Cells in Mechanoregulation. Cellular and Biomolecular Mechanics and Mechanobiology, A. Gefen, ed., Springer, 04, 295-329. Springer. doi:10.1007/8415

McLoughlin, R. (2008). Tensile Fatigue Behaviour of Porcine Coronary Arterial Tissue in the Longitudinal and Circumferential Directions. MSc Thesis. University of Dublin.

Pérez, M. a, \& Prendergast, P. J. (2007). Random-walk models of cell dispersal included in mechanobiological simulations of tissue differentiation. Journal of biomechanics, 40(10), 224453. doi:10.1016/j.jbiomech.2006.10.020

Thyberg, J., Hedin, U., Sjolund, M., Palmberg, L., \& Bottger, B. A. (1990). Regulation of differentiated properties and proliferation of arterial smooth muscle cells. Arteriosclerosis, Thrombosis, and Vascular Biology, 10(6), 966. Am Heart Assoc.

Timmins, L.H., Moreno, M. R., Meyer, C. A., Criscione, J. C., Rachev, A., \& Moore, J. E. (2007). Stented artery biomechanics and device design optimization. Medical and Biological Engineering and Computing, 45(5), 505-513. Springer. doi:10.1007/s11517-007-0180-3

Welt, F., \& Rogers, C. (2002). Inflammation and restenosis in the stent era. Arteriosclerosis, Thrombosis, and Vascular Biology, 22, 1769-1776.

Windecker, S., \& Meier, B. (2001). All stents are not alike or is the difference in the eye of the observer only? European Heart Journal, 22(21), 1973-7. doi:10.1053/euhj.2001.2847 


\section{Figures}

\section{MULTILINK}
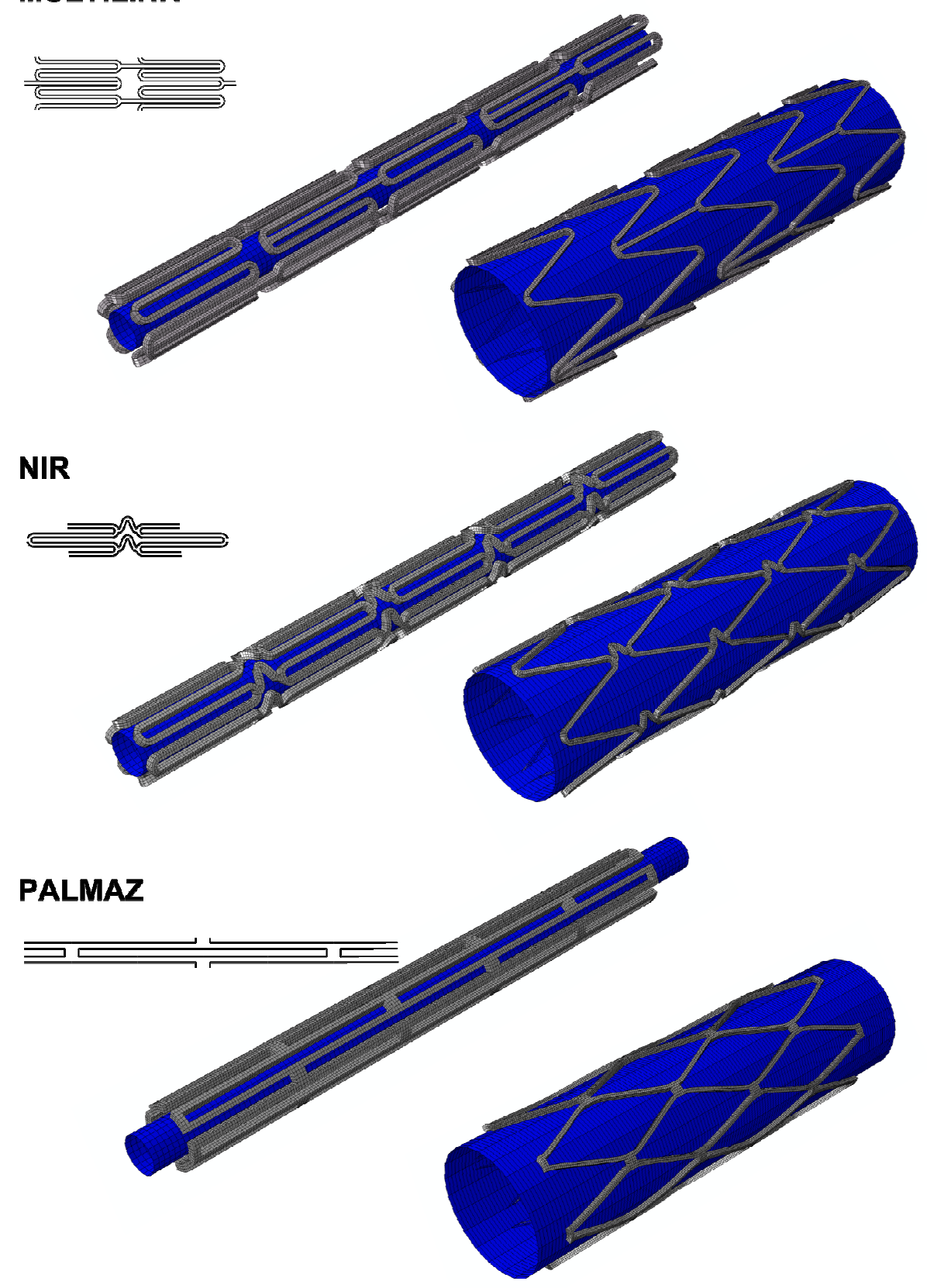

Figure 1. The three stents simulated. Each stent shows the unexpanded configuration and the stent at maximum expansion, as well as a $2 \mathrm{D}$ representation of the repeating unit that makes up the stent. 
MULTILINK

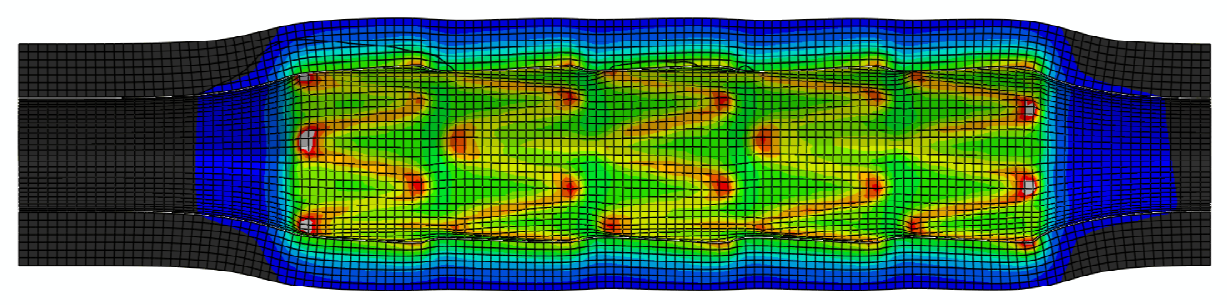

von Mises stress (MPa)

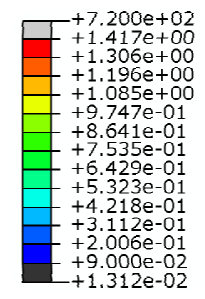

NIR

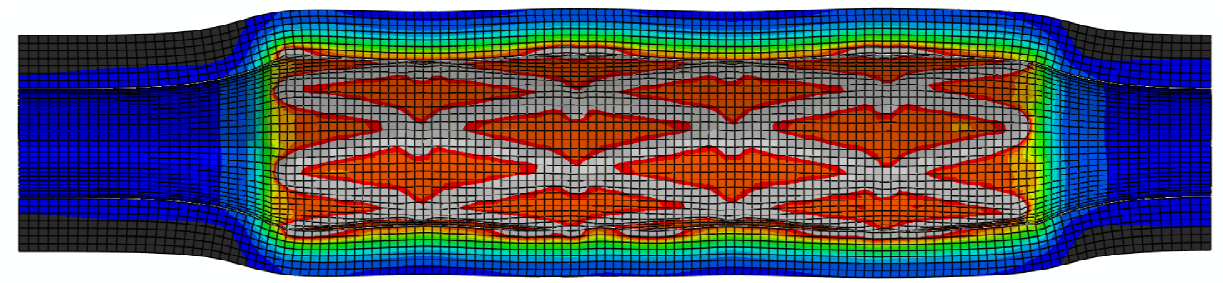

PALMAZ

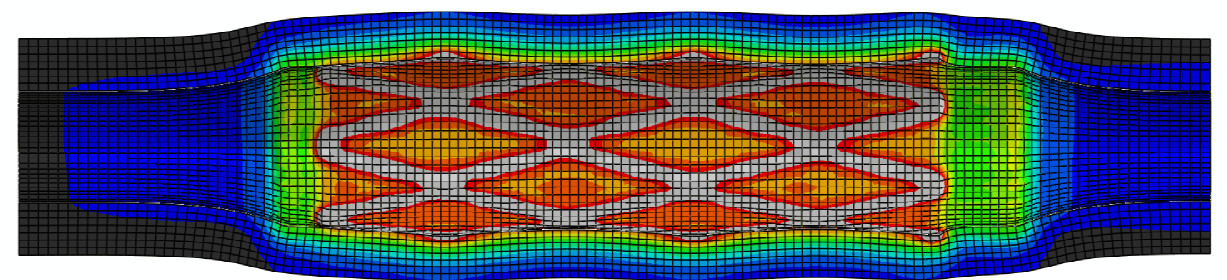

Figure 2. Contour plot of von Mises stress (in MPa) at $80 \%$ expansion indicating the areas above the maximum injury, i.e. greater than failure strength (1.417 MPa) in light grey. The dark grey regions indicate regions of tissue below the fatigue strength of the tissue (0.09 $\mathrm{MPa})$. 


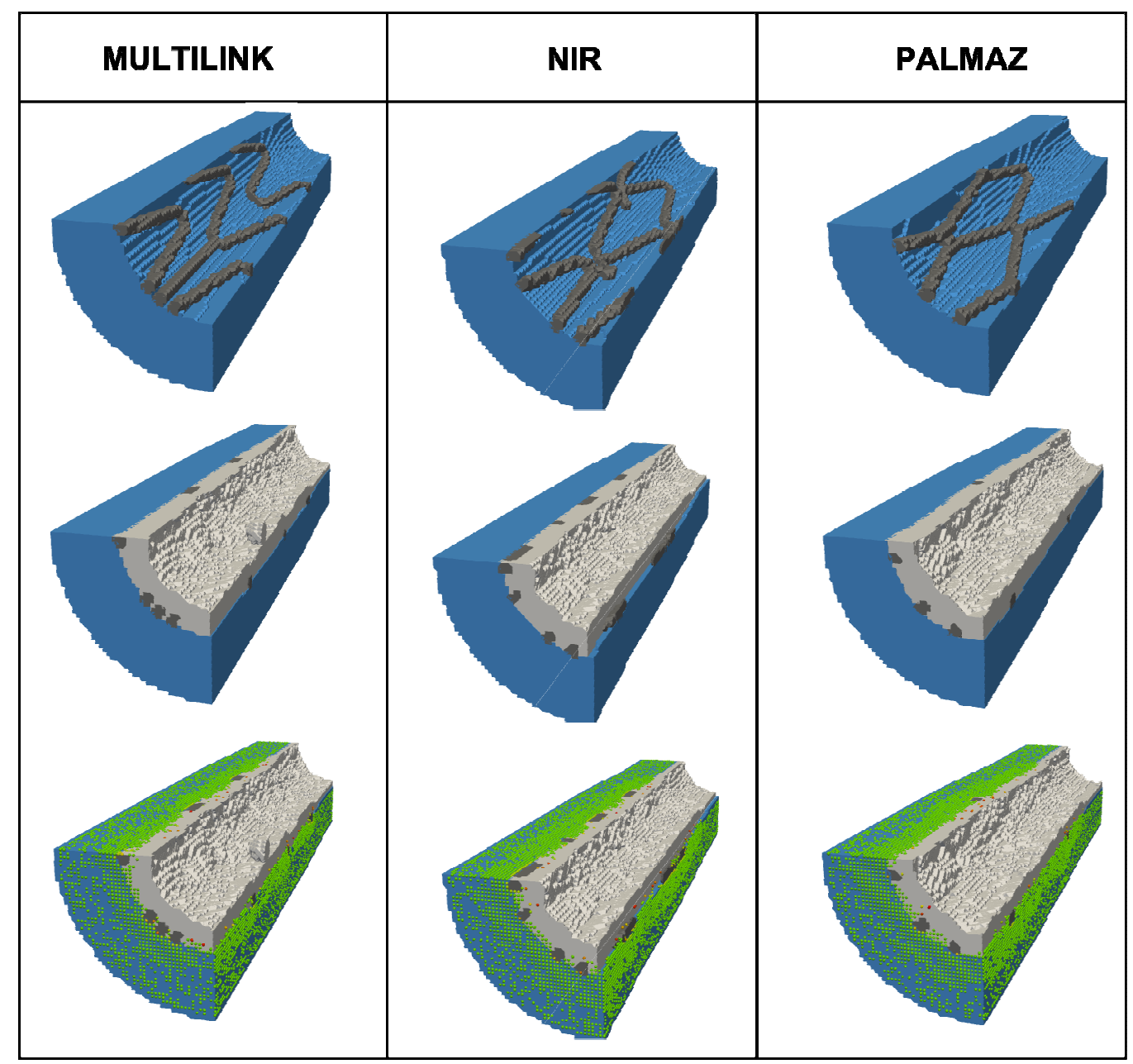

\section{Media Tissue Stent Material $\square$ Neointima}

\section{Smooth Muscle Cells 0000 synthetic $\longleftrightarrow$ contractile Phenotype}

Figure 3. A plot of the lattice models showing $1 / 8$ of the full model. Above, the lattice representation of the artery and stent immediately post-stenting. Centre, showing the neointimal volume superimposed on the original geometry. Bottom, the lattice with SMCs at the end point of the simulation. 


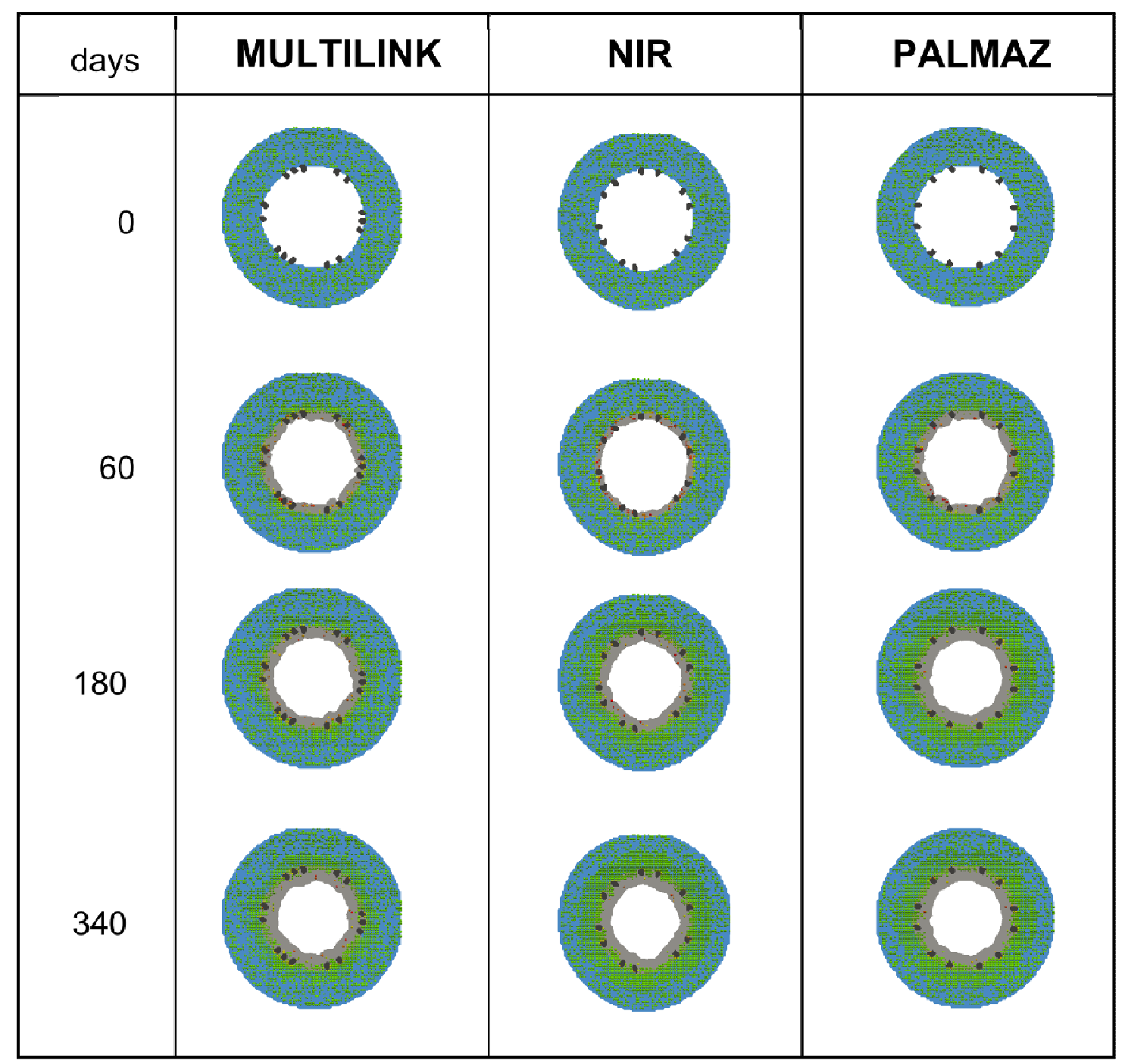

Figure 4. Simulation plot of the lattice over time in the simulation of all stents, a section of a z-normal plane at the midsection of the stent. This plot shows the distribution and phenotype of cells. Neointima is in grey. 


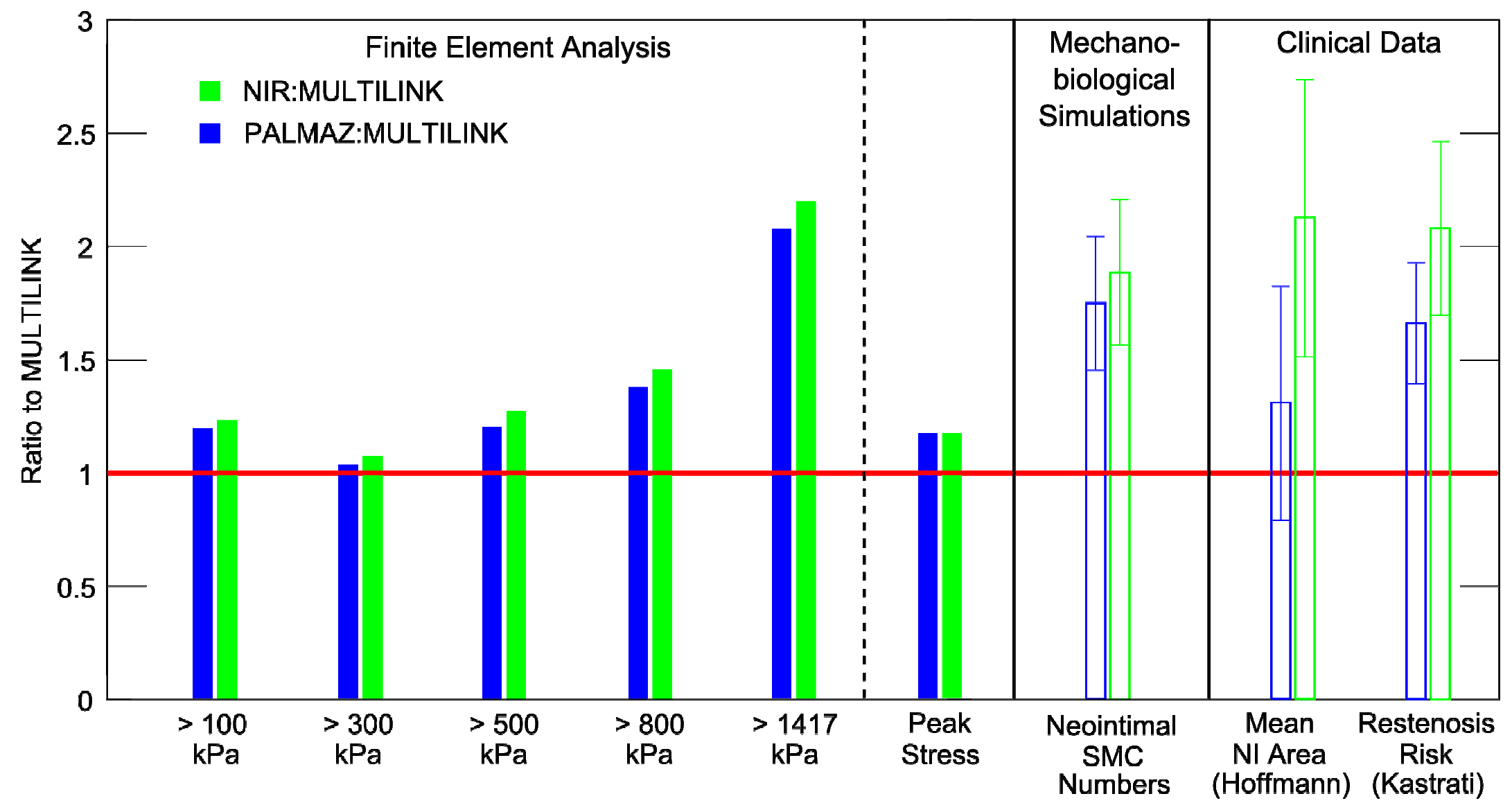

Figure 5. The relative performance of the three stents based on several different criteria. The PALMAZ and NIR stents are given as ratios to the best performing (MULTILINK) stent. The first five are the volume of arterial tissue above a critical stress. The sixth criterion is the peak stress induced in the artery. The seventh criterion is based on the numbers of cells in the neointima measured in the simulations for all inflammation levels. The eighth criterion is the mean neointimal area measured by Hoffmann et al. (2001), including standard deviation. The ninth criterion is the risk of restenosis, judged through multivariate analysis of binary restenosis rates (Kastrati et al., 2001 ).. Error bars are \pm 1 S.D. 

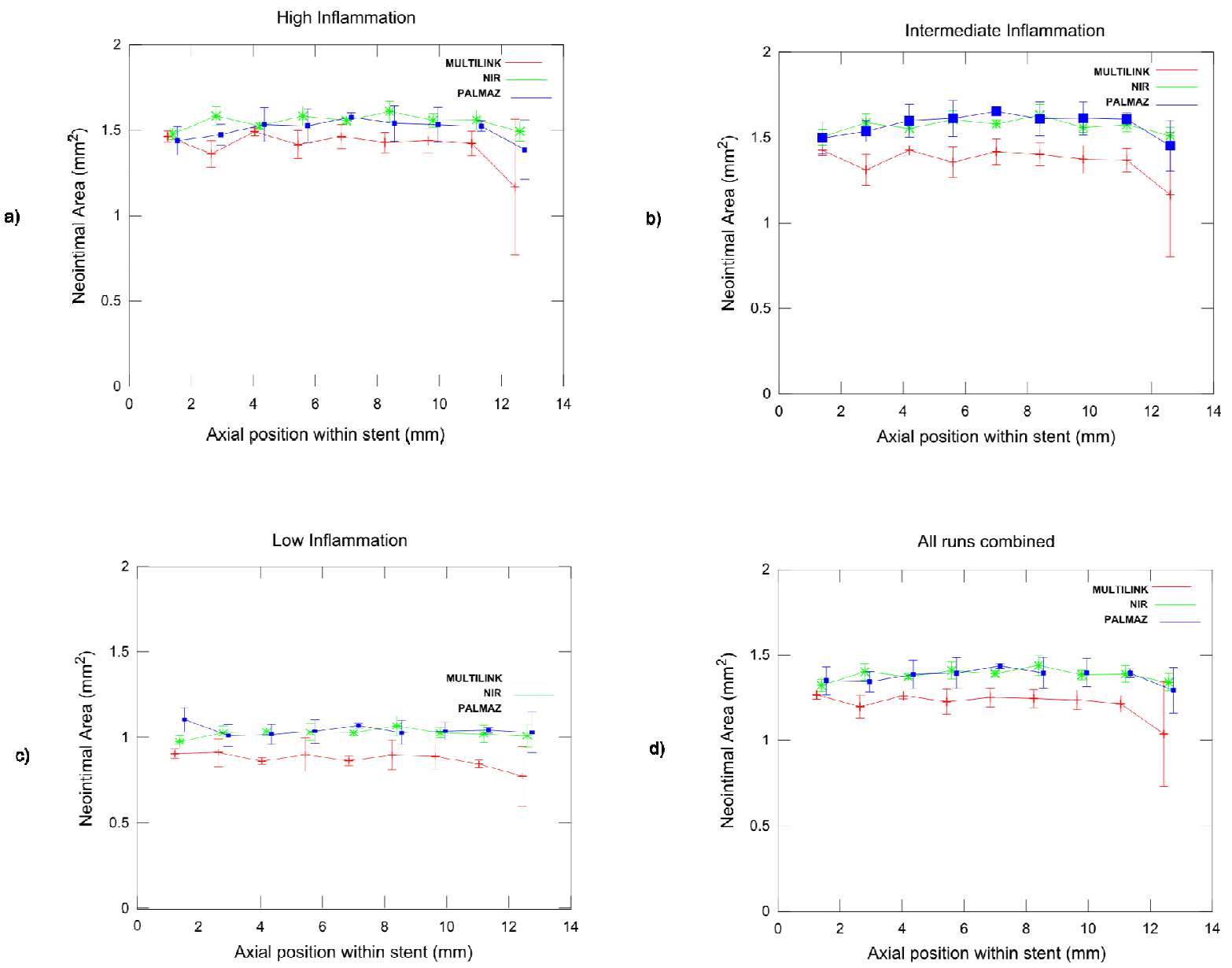

Figure 6. The cross sectional area of restenosis within the stents along the axial direction. This diagram combines all data from high, low and intermediate inflammation for each stent. 


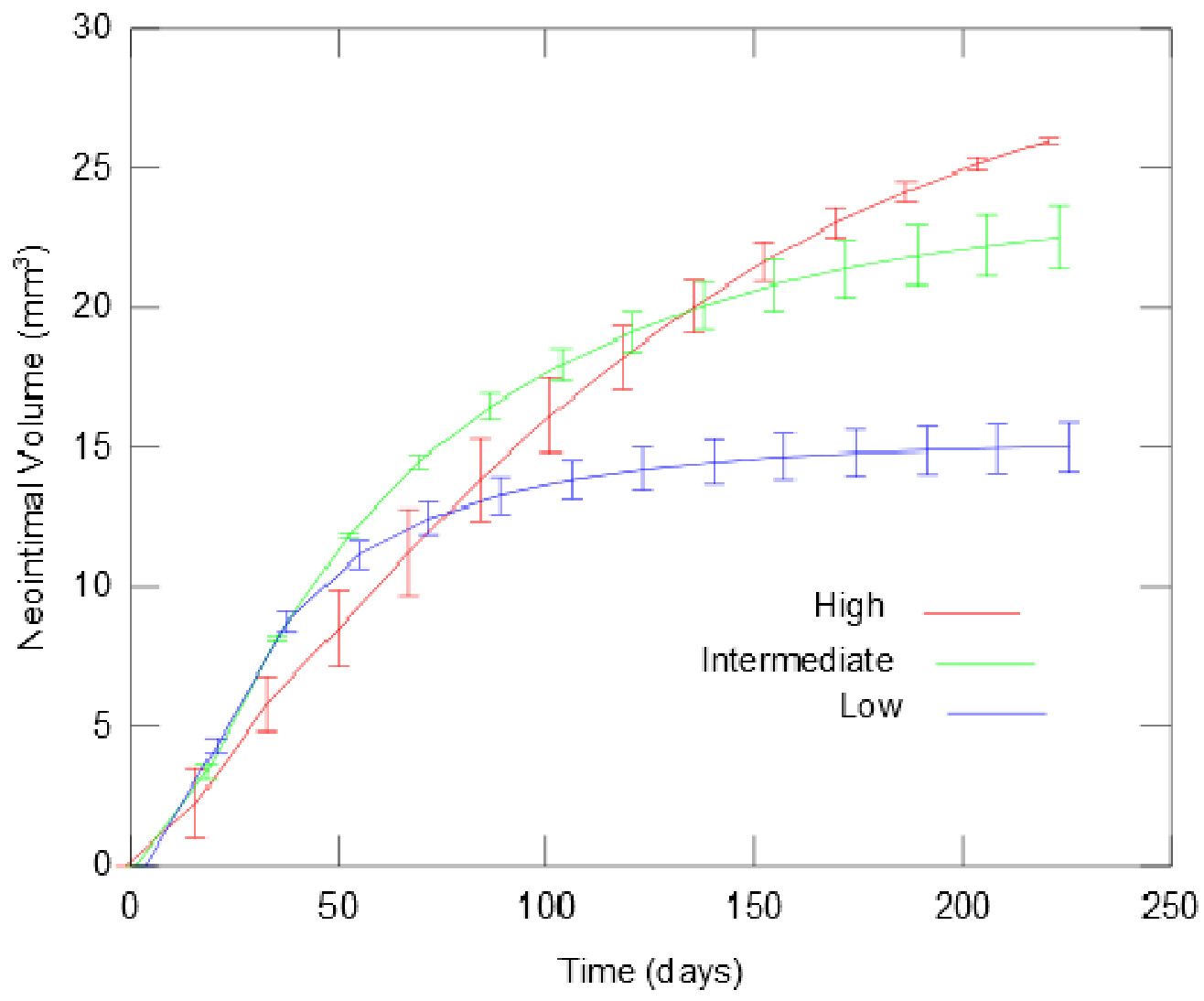

Figure 7. Volume of neointima within the MULTILINK stent over time for the three inflammation rates. Similar trends were found for the PALMAZ and NIR stents (not shown). 

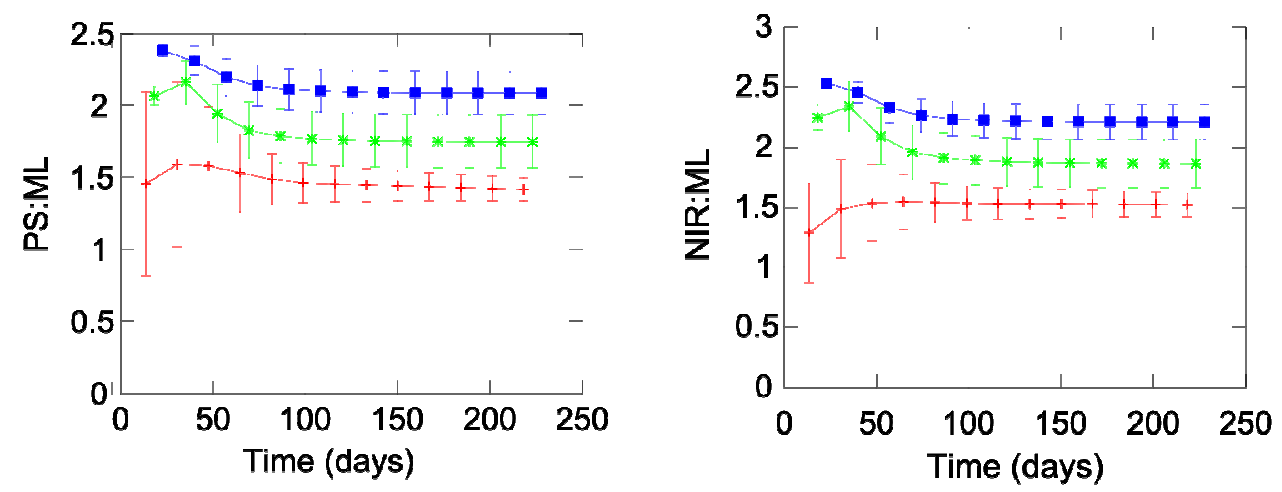

Inflammation:

- High
Intermediate

Low

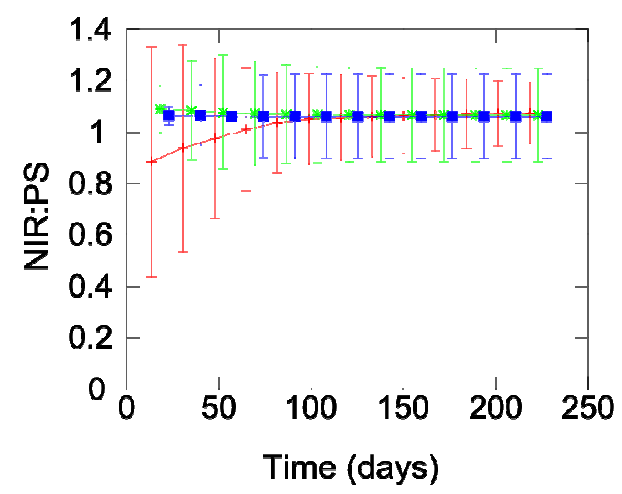

Figure 8. A plot of the ratio of the amounts of neointimal cells between the three stents. The numbers of neointimal SMCs in the model was calculated for each stent. The ratio of the numbers of cells produced between stents is plotted here for each of the stent combinations. A value of 1 indicated no difference between the stents. 